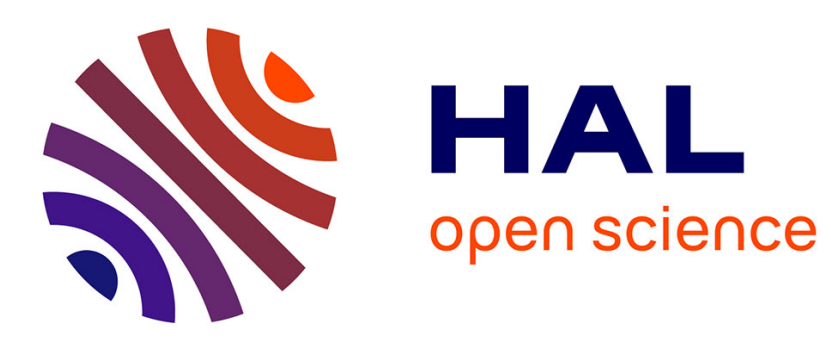

\title{
Construire l'auto-efficacité par l'analyse de l'activité en formation des cadres et dirigeants de la santé publique Marc Nagels
}

\section{To cite this version:}

Marc Nagels. Construire l'auto-efficacité par l'analyse de l'activité en formation des cadres et dirigeants de la santé publique. Savoirs: Revue internationale de recherches en éducation et formation des adultes, 2010, 2010/1 (22), pp.69 - 88. hal-00516291

\section{HAL Id: hal-00516291 \\ https://hal.science/hal-00516291}

Submitted on 9 Sep 2010

HAL is a multi-disciplinary open access archive for the deposit and dissemination of scientific research documents, whether they are published or not. The documents may come from teaching and research institutions in France or abroad, or from public or private research centers.
L'archive ouverte pluridisciplinaire HAL, est destinée au dépôt et à la diffusion de documents scientifiques de niveau recherche, publiés ou non, émanant des établissements d'enseignement et de recherche français ou étrangers, des laboratoires publics ou privés. 


\section{Construire l'auto-efficacité par l'analyse de l'activité en formation des cadres et dirigeants de la santé publique}

L'objet de cette recherche était de mesurer la contribution de l'analyse de l'activité au développement de l'auto-efficacité au travail en référence à la théorie sociocognitive et à la théorie de la conceptualisation dans l'action. Notre population est constituée de cadres et de dirigeants de la santé publique en formation à l'École des hautes études en santé publique. Un dispositif d'autoconfrontations a été organisé pour évaluer la corrélation entre l'analyse réflexive de son activité et le niveau d'auto-efficacité. L'auto-efficacité des élèves a été mesurée prétest et posttest. Après vérification statistique, nous observons que l'analyse de l'activité a eu un effet sur le groupe test et, plus précisément, sur les personnes de faible niveau initial d'auto-efficacité. Nous en concluons que l'analyse de l'activité et l'agentivité contribuent explicitement à la formation des compétences.

Mots-clés : Compétence, auto-efficacité, analyse de l'activité, directeur des soins.

\section{Build the self-efficacy by the activity analysis in training of managers and leaders of the public health}

The aim of this research is to measure the contribution of the activity analysis theory to the self-efficacy development at work related to the socio-cognitive theory and to the conceptualization in action theory. Our population is made up of executives and leaders of public health training in the French school of public health in Rennes, France. A plan of selfconfrontations has been organized in order to evaluate the correlation between the reflexive analysis of their activity and their level of self-efficacy. The student self-efficacy was measured pre test and post test. On statistics checking, an effect on the testing group has been observed after their activity analysis and more distinctly for the students with an initial low level of self-efficacy. We can conclude that activity analysis and agentivity explicitly contribute to the competencies training.

Key words : competencies, self-efficacy, activity analysis, director of nursing 


\section{Construir la autoeficacia para el análisis de la actividad en formación de los marcos ejecutivos y dirigentes de la Salud Pública}

El objeto de esta investigación fue medir la contribución del análisis de la actividad al desarrollo de la autoeficacia del trabajo en referencia a la teoría sociocognitiva y a la teoría de la conceptualización en la acción. Nuestra población está constituida por marcos ejecutivos y dirigentes de la Salud Pública en formación en la Escuela de estudios profesionales en Salud Pública. Un dispositivo de autoconfrontaciones fue organizado para evaluar la correlación entre el análisis reflexivo de su actividad y el nivel de autoeficacia. La autoeficacia de los alumnos fue medida antes y después de la prueba. Después de comprobación estadística, observamos que el análisis de la actividad tuvo un efecto sobre el grupo evaluado, más precisamente, sobre las personas de nivel inicial de autoeficacia. Concluimos que el análisis de la actividad explícitamente contribuye a la formación competitiva.

Palabras clave: competencia, autoeficacia, análisis de la actividad...

\section{Construire l'auto-efficacité au travail par l'analyse de l'activité en formation des cadres et dirigeants de la santé publique.}

\section{Introduction}

En ces temps de pandémie grippale, les usagers du système de santé que nous sommes observent avec beaucoup d'attention l'activité des cadres et des dirigeants de la santé publique. Leurs responsabilités sont considérables pour décider des stratégies de prévention, prévoir les plans de lutte contre la propagation du virus, communiquer sur les mesures de prophylaxie et organiser l'accueil et le traitement des personnes atteintes. Les cadres et 
dirigeants sont mobilisés pour manager les services de l'État et les centres hospitaliers et faire fonctionner le système de santé dont nous bénéficions. D’une manière générale, outre la diffusion mondiale rapide des pathologies, les dirigeants de la santé publique ont à se préoccuper de problématiques liées au vieillissement des populations, à la prise en charge des personnes atteintes de maladies chroniques, aux crises sanitaires ou encore aux déterminants de santé telles que les inégalités ou les injustices sociales «qui tuent à grande échelle », comme le rappelle sans cesse l'Organisation mondiale de la santé. Leurs compétences sont nombreuses et visent à répondre à des situations critiques de nature très différentes.

L'École des hautes études en santé publique (EHESP) est l'établissement français de formation supérieure professionnelle qui forme les cadres et les dirigeants de la santé publique. L'école accueille des élèves en formation statutaire en vue d'exercer trois types de métiers : dirigeants d'établissements sanitaires et sociaux (directeurs d'hôpital, directeurs des soins, etc.), régulateurs de l'action sanitaire et sociale publique (médecins et pharmaciens inspecteurs, inspecteurs des affaires sanitaires et sociales, etc.), experts de la santé environnementale, par exemple des ingénieurs du génie sanitaire. Le public en formation statuaire est souvent titulaire d'un master ou d'un doctorat mais l'école accueille de nouvelles populations et délivre dorénavant six masters en propre ou en co-habilitation. Le réseau doctoral est en cours de consolidation.

La recherche et les résultats qui vont être exposés dans cet article s'insèrent dans cette période de mutation, où l'ancienne École nationale de santé publique vient d'acquérir le statut de « grand établissement » et où la finalité de la formation des compétences n'a pas cédé devant un impératif plus académique de transmission de savoirs. Les orientations institutionnelles ne privilégient pas le développement des formations académiques au détriment de la formation des compétences professionnelles, et ceci en pleine conformité avec les orientations de l'enseignement supérieur européen.

\section{Problématique}

Dans ce contexte particulier, les pratiques de formation de l'EHESP peuvent toujours être questionnées: l'objectif général de formation des compétences s'accompagne-t-il systématiquement d'objectifs pédagogiques pertinents et relatifs aux ressources à mobiliser 
pour agir efficacement? Les intentions et les pratiques pédagogiques observables prennentelles en compte les situations professionnelles de référence sans en rester au contexte légal et réglementaire et aux enjeux de santé publique ? Ce sont aussi les traits culturels et les pratiques hospitalières qui permettent $d$ 'apprécier la performance et de comprendre l'évaluation qui en est faite dans les services. Par ailleurs, les situations d'apprentissage intègrent-elles largement les ressources cognitives et environnementales, les stratégies comportementales, que les apprenants peuvent mobiliser pour réussir et dépasser les obstacles?

Nous avons voulu en savoir plus sur ce lien étroit entre les situations de travail en milieu social et sanitaire et l'activité du professionnel novice. Nous nous sommes situé délibérément du point de vue du sujet social apprenant et non du dispositif de formation. En effet, un dispositif de formation, le meilleur soit-il, n'est jamais qu'une offre de formation, qu'une occasion de développement professionnel présentée à l'apprenant (MÉTRAL J-F., MAYEN P., 2007). Il doit s'articuler à la motivation, aux capacités cognitives de l'apprenant et à sa résilience dans la découverte du terrain professionnel (JÉZEGOU A., 2005). Cette recherche interroge ainsi les déterminants internes du sujet social apprenant tels que l'auto-efficacité au travail et l'analyse de l'activité, source de développement possible de l'auto-efficacité. L'autoefficacité est définie comme «la croyance de l'individu en sa capacité d'organiser et d'exécuter la ligne de conduite requise pour produire des résultats souhaités » (BANDURA A., 2003). Analyser l'activité conduit, par ailleurs, à considérer le sujet qui l'exerce, mais aussi la tâche à laquelle elle répond et l'ensemble des conditions techniques, sociales et organisationnelles qui la constitue (LEPLAT J., 2000).

Plusieurs recherches sur la formation des compétences des apprenants de l'EHESP ont été conduites (COULET J-C., CHAUVIGNÉ C., 2005; NAGELS M., POURRIERE J-L., 2007). Ces recherches prennent appui souvent sur une problématique de l'activité et de l'organisation cognitive de l'activité. L'usage des référentiels de compétences, même et surtout quand ils ne se limitent pas à une liste de tâches prescrites mais analysent l'activité réelle et proposent la formulation de la structure conceptuelle de la situation, a été exploré sous l'angle de la théorie instrumentale (NAGELS M., LE GOFF M., 2008).

Ces recherches sont sous-tendues par un modèle de la compétence qui articule une dimension interne et une dimension externe. La dimension interne au sujet est celle d'un sujet capable 
(RABARDEL P., 2005), auto-efficace pour ses apprentissages professionnels (BANDURA A., 2005; FRANÇOIS P-H., BOTTEMAN A., 2002) qui analyse sa propre activité et qui apprend dans les situations et par les situations (PASTRÉ P., MAYEN P., VERGNAUD G., 2006). La dimension externe est constituée des processus d'évaluation de la performance par l'environnement social et professionnel mais aussi des modes de réaction qu'ils génèrent. En situation de management hospitalier par exemple, l'apprenant est évalué en permanence. Sa performance est examinée sous l'angle de la transformation du travail d'autrui en performance (GALAMBAUD B., 2002) et la réussite est appréciée selon les choix de l'organisation (ENLART S., 2007; ENLART S., 2008). La compétence, puisqu'elle est évaluation, est une affaire de normes, de référentiels, et donc aussi de déviances et d'écarts à la norme. L'accent est mis sur la production de comportements conformes aux attentes sociales. Les comportements peuvent ainsi obéir à d'autres raisons que les motivations internes de l'individu. Ils peuvent également être provoqués par des déterminations externes. En matière de compétence aussi, «seuls les actes nous engagent. Nous ne sommes donc pas engagés par nos idées, ou par nos sentiments, mais par nos conduites effectives » (BEAUVOIS J-L., JOULE R-V., 1987). L'engagement est défini par le lien qui existe entre un individu et ses comportements (KIESLER C., 1971). En retour, les individus sont amenés à ruser, à mettre en œuvre des stratégies de contournement et d'évitement. Le succès de ces stratégies de protection face aux tentatives de normalisation de l'institution repose sur l'usage de la clairvoyance normative : «la connaissance (...) du caractère normatif ou contrenormatif d'un type de comportements sociaux, ou d'un type de jugements, et d'autre part, de la conformité ou de la non-conformité d'un comportement par rapport à ce qui est attendu par un individu possédant un certain statut » (PY J., SOMAT A., 1991). Les individus décryptent mieux leur environnement et ils décèlent plus efficacement les attentes et injonctions professionnelles.

La figure suivante synthétise les différentes composantes d'un modèle agentique de la compétence. 
Figure 1 : Dimensions internes et externes de la compétence

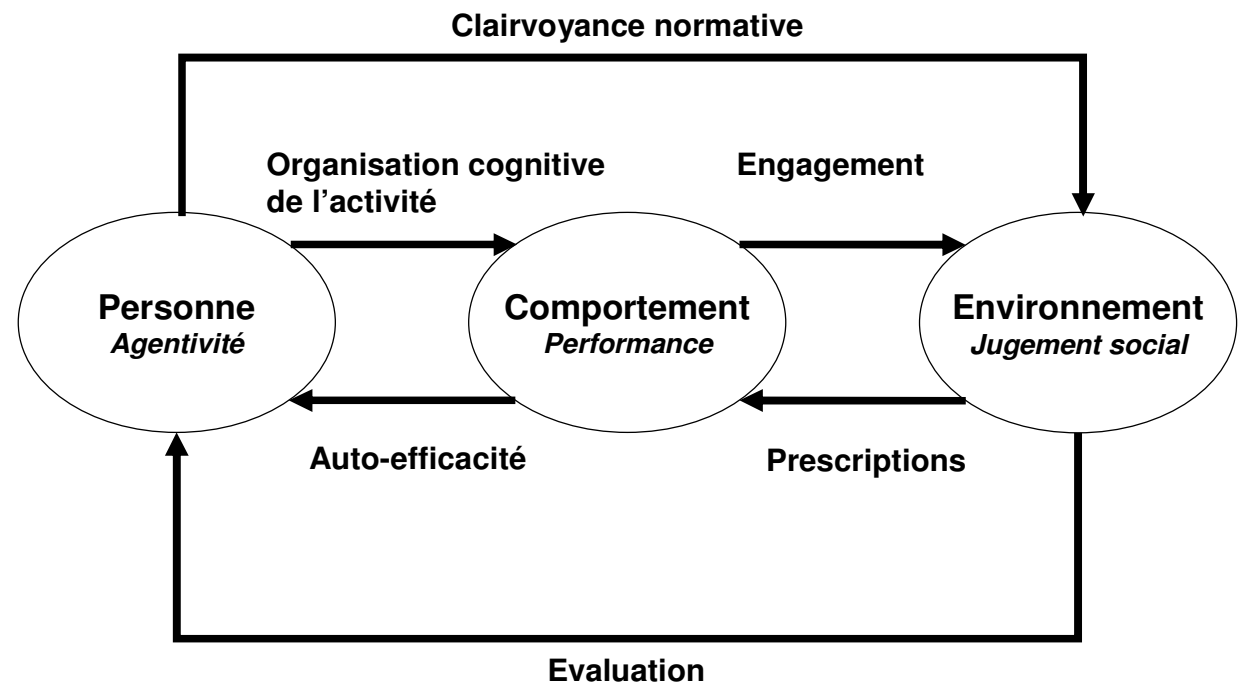

La compétence s'inscrit dans une perspective de construction et de renforcement de l'agentivité humaine, c'est-à-dire de la capacité d'intervention sur les autres et le monde. L'agentivité se manifeste de trois manières différentes : il s'agit tout d'abord de l'effet direct de l'intervention de la personne mais elle peut opérer aussi par le biais d'une «procuration » par laquelle le sujet escompte l'action d'autrui pour atteindre les buts souhaités. Enfin, l'agentivité peut être collective, par l'intermédiaire d'une coordination des efforts d'un groupe (BANDURA A., 2003).

Nous le voyons dans ce modèle agentique de la compétence, l'analyse de l'activité et l'autoefficacité peuvent se conjuguer pour produire la performance mais expliquent aussi, pour partie, les comportements individuels de conformité, ou de prise de risque, des apprenants face à l'évaluation de leur performance. Il est permis de supposer que ces deux processus sont en interaction et se renforcent mutuellement dès lors que l'apprentissage est complexe : acquérir des compétences de haut niveau et gérer des problèmes mettant en jeu une forte responsabilité et des décisions stratégiques pour la mise en œuvre du système de santé. Concevoir que l'analyse de l'activité et l'auto-efficacité peuvent interagir pour un apprenant n'est pas se prononcer sur le type d'interaction. C'est pourquoi, nous avons voulu tester l'hypothèse suivante: inscrire les apprenants dans un processus d'analyse de l'activité augmenterait le niveau d'auto-efficacité au travail. Cette hypothèse est nouvelle et représente 
une avancée au sein de la théorie sociocognitive puisque l'analyse de l'activité n'est pas une source de l'auto-efficacité explicitement prévue.

\section{Méthodes}

La collecte des données s'est déroulée en trois phases successives.

Premièrement, des entretiens exploratoires semi-directifs ont été conduits auprès d'un échantillon de médecins inspecteurs de santé publique et de directeurs des soins pour repérer les compétences perçues et les apprentissages à réaliser. Le niveau d'auto-efficacité au travail pour ces métiers a aussi été interrogé. Les transcriptions d'entretiens ont fait l'objet d'une analyse thématique de contenu.

Deuxièmement, le niveau d'auto-efficacité a été mesuré auprès de 697 élèves de l'EHESP à l'aide d'une échelle de mesure spécifique (FOLLENFANT A., MEYER T., 2003). L'échelle d'auto-efficacité est une échelle de type Likert. Elle comprend dix énoncés de forme déclarative orientés positivement, se référant à des comportements spécifiques en milieu professionnel, par exemple : «Dans mon travail, je parviens toujours à résoudre les problèmes difficiles si je m'en donne la peine ». Ils sont suivis de réponses indiquant plusieurs niveaux d'accord possibles : « tout à fait d'accord », «plutôt d'accord », « pas vraiment d'accord », « pas du tout d'accord ». Une seule réponse est possible par énoncé et il n'y a pas de point milieu. Le score minimal est de dix points et le score maximal est de quarante points pour cette échelle. Le calcul du score moyen par filière de formation peut être obtenu aisément par la suite. Les résultats principaux portent ainsi sur le score d'auto-efficacité mis en classes et sur le score moyen obtenu par chaque filière de formation. Nous avons ainsi évalué des capacités et non de simples intentions à l'aide de statistiques descriptives qui permettent de représenter et de quantifier la variabilité des grandeurs observées.

Troisièmement, une expérimentation visait à tester si la participation à un dispositif d'analyse de l'activité de décision en management hospitalier favorisait le développement de l'autoefficacité au travail. Cette expérimentation a été conduite auprès de la promotion des directeurs des soins en constituant un groupe test de 18 participants et un groupe contrôle de 48 personnes. Le test de Wilcoxon, test de symétrie d'une variable par rapport à une valeur donnée, a été utilisé pour se prononcer sur les effets de l'analyse de l'activité. Quelques 
entretiens ont ensuite complété le dispositif pour mieux comprendre, d'un point de vue plus qualitatif, les effets produits par l'analyse de l'activité sur l'auto-efficacité.

Le dispositif empirique est essentiellement basé sur les autoconfrontations pour rendre observable les dimensions de l'activité qui ne le sont pas aisément la plupart du temps (CLOT Y., FAÏTA D., FERNANDEZ G., SCHELLER L., 2000; FABRE M., 2006). Dans e cadre, e debriefing (PASTRÉ P., 2005a) se présente comme une méthode d'analyse de l'activité a posteriori, le debriefing reposant lui-même sur un mode d'action spécifique : la reconstruction de l'intrigue (PASTRÉ P., 2005b; RICEEUR P., 1986).

La première phase consiste en une auto-analyse en appui sur des traces de l'activité de décision, quelle que soit la nature de ces traces. La phase d'auto-analyse fait l'objet d'un écrit descriptif du contexte professionnel de l'action, de la décision prise, du but poursuivi, des paramètres situationnels pris en compte pour décider, des savoirs de type académique et des savoirs de type pragmatique, des résultats souhaités et des phases d'élaboration de la décision. L'écriture du texte sur la décision est un temps d' «auto-analyse » (OLRY P., 2004; SIXTOUCHARD B., 1998; SIX-TOUCHARD B., CARLIN N., 2003), l'auto-analyse du travail consistant à matérialiser une trace des conceptualisations produites lors de l'activité. Du moins c'est l'objectif visé, il ne peut être atteint que partiellement puisque ces auto-analyses contiennent aussi des discours argumentatifs portant sur soi en situation. Faire produire des conceptualisations relève de la suite du processus, processus guidé par le chercheur.

Lors de la deuxième phase, neuf binômes de directeurs des soins ont été constitués à l'intérieur du groupe test : trois binômes d'élèves de niveau élevé d'auto-efficacité (de 35 à 38 points, score moyen égal à 36,67 avec un écart-type de 1,12), trois binômes de niveau bas, trois binômes mixant un élève de niveau élevé et un élève de niveau bas (de 24 à 27 points, score moyen égal à 25,67 avec un écart-type de 1,22). La répartition dans les binômes est décidée par un tiers sans que les scores d'auto-efficacité ne soient communiqués pendant le temps de l'expérimentation, ni au chercheur ni aux intéressés. Le groupe test est donc formé de deux sous-groupes : niveau initial d'auto-efficacité élevé et niveau initial d'auto-efficacité faible. Le groupe contrôle, presque les trois-quarts de la promotion, soit 48 personnes, peut être décrit sur les mêmes critères : quinze personnes obtiennent un niveau d'auto-efficacité élevé (de 35 à 38 points, score moyen de 33,33 points avec un écart-type de 0,98), onze personnes présentent un niveau d'auto-efficacité faible (de 24 à 28 points, score moyen de 
27,18 points avec un écart-type de 1,17). De plus, entre ces deux sous-groupes contrôle, émerge un sous-groupe de niveau d'auto-efficacité intermédiaire (vingt-deux personnes de 29 à 31 points, score moyen de 29,92 points avec un écart-type de 0,72 ). Le groupe contrôle est donc constitué de trois sous-groupes : niveau élevé, niveau intermédiaire et niveau faible. Chacun de ces sous-groupes, test et contrôle, est donc assez homogène, l'écart-type portant sur les scores restant faible.

La troisième phase est celle des autoconfrontations croisées au sein de chaque binôme, d'une durée moyenne chacune de quarante-cinq minutes. Le matériel support est celui de la première phase : traces de l'activité et écrit préalable du sujet. L'analyse se déroule entre les deux apprenants en présence du chercheur. Il s'agit de recueillir les éléments constitutifs du modèle cognitif et du modèle opératif du sujet, les savoirs et les concepts organisateurs.

La dernière phase est la rencontre entre tous les binômes, organisée et animée par le chercheur, pour présenter et discuter des buts poursuivis dans l'activité, des concepts organisateurs et des savoirs utilisés en situation de décision.

Une mesure d'auto-efficacité était effectuée à la fin du dispositif d'analyse de l'activité et pouvait être comparée à la mesure pré test.

\section{Résultats}

Dans un premier temps, l'analyse des entretiens exploratoires a permis d'identifier un ensemble de compétences, telles qu'elles sont citées spontanément et commentées abondamment par les médecins inspecteurs de santé publique et les directeurs des soins. Ces compétences sont présentées comme signant l'appartenance au métier. Avant tout, ce sont des compétences managériales, relationnelles, organisationnelles, c'est-à-dire des « compétences sociales et relationnelles » et qu'il est parfois risqué de mettre en relation avec des traits de personnalité (THIBERGE B., 2007) ou même de vouloir les évaluer. En santé publique, les compétences sociales managériales peuvent faire facilement défaut aux professionnels novices dans leur nouvelle fonction, et, contrairement aux compétences «techniques », elles requièrent une forte implication relationnelle dans des situations délicates où les arbitrages sont lourds de conséquences. 
Les participants qui ont été interrogés expliquent que les novices ne possèdent pas toujours ces compétences mais qu'elles sont pourtant indispensables pour exercer le métier. Décrites ainsi, ces compétences deviennent des compétences «critiques », c'est-à-dire des «compétences acquises par un individu au cours de son expérience, et qui font de lui quelqu'un d'irremplaçable dans certaines tâches (...) Le caractère critique d'une compétence ne se mesure pas seulement au caractère difficilement remplaçable de l'individu qui la possède mais aussi à la difficulté qu'il y a à l'acquérir »(VERGNAUD G., 1998). C'est pourquoi tous les professionnels n'en disposent pas et tout particulièrement les professionnels novices. Les compétences critiques représentent un enjeu de la professionnalisation des individus. Elles font la différence entre les individus et entre les métiers.

La compétence critique présenterait cette caractéristique de ne pas immerger l'individu trop rapidement dans l'action, de conserver une certaine distance avec la prescription et de lui faire choisir le mode de résolution de problèmes le mieux adapté. L'individu privilégie la prise d'information à caractère diagnostique pour mieux réguler son action en fonction de ses buts et sous-buts. L'effet produit consiste en l'adaptation optimum à l'environnement et aux variables situationnelles à prendre compte. L'acquisition de compétences critiques se traduit par des schèmes qui s'appliquent à des classes de situations étendues mais aussi par la mobilisation d'inférences plus nombreuses, de meilleures représentations des buts et des résultats intermédiaires.

Néanmoins, nous allons considérer pour la suite de la recherche que ces compétences, pour critiques qu'elles soient, ne sont après tout que des compétences et qu'elles sont analysables avec les mêmes outils. Nous pouvons décrire les compétences en mettant en lumière les schèmes (VERGNAUD G., 2006) que les individus mobilisent. Les schèmes des compétences critiques présentent un haut degré d'organisation des invariants opératoires, organisation hiérarchisée à l'intérieur d'un réseau de concepts-en-acte et de théorèmes-en-acte. Les invariants opératoires, se rapprochent des «concepts organisateurs » de la structure conceptuelle de la situation (PASTRÉ P., MAYEN P., VERGNAUD G., 2006) et peuvent rester largement implicites.

Les médecins inspecteurs de santé publique et les directeurs des soins n'exercent pas sur les mêmes terrains et donc ne désignent pas les mêmes compétences critiques. Les médecins inspecteurs de santé publique, déclarent tous, sans exception, que les compétences de 
planification des actions, de gestion des priorités, de mobilisation des services et des partenaires sont critiques.

Ce noyau de compétences sociales et relationnelles est partagé par les directeurs des soins. En outre, ceux-ci considèrent unanimement en entretien, que leur travail consiste à résoudre les difficultés de management des équipes et à agir pour optimiser l'activité des services face aux contraintes hospitalières en rapide évolution. Les directeurs des soins désignent explicitement des compétences d'autoréflexivité et d'analyse de leurs comportements.

Il apparaît toutefois que si les compétences sont comparables entre les deux métiers, les niveaux d'auto-efficacité diffèrent selon l'origine professionnelle. La plupart des médecins inspecteurs de santé publique expriment qu'ils traitent de manière peu satisfaisante les problèmes professionnels qui se présentent : «Comment puis-je gérer tout cela ? Je ne sais pas. Je pense que j'ai beaucoup de difficulté ». Ils subissent souvent les situations sans toujours trouver les solutions aux problèmes. Considérer que les obstacles qui se présentent ne peuvent être dépassés est l'indice d'un faible niveau de compétences perçues.

Les directeurs des soins expriment un plus haut niveau d'auto-efficacité. Deux indicateurs en témoignent : ils estiment réussir plus fréquemment à identifier les situations sur lesquelles agir et pensent mieux piloter les adaptations de l'environnement hospitalier. Pour eux, la réflexivité tient une grande place dans l'acquisition des compétences critiques et ils cherchent à la développer au sein de leurs équipes de cadres. L'un d'entre eux s'exprime ainsi : «c'est une fonction qui doit avoir une force d'écoute, d'analyse et de communication pour arriver toujours à un consensus. J'ai douze cadres supérieurs, dont dix en secteur clinique et deux en missions transverses. Je n'ai pas d'autres solutions que d'expliquer et de faire en sorte que mes cadres supérieurs soient imprégnés de cette nouvelle culture, qu'ils trouvent eux aussi des repères et qu'ils cherchent des organisations nouvelles pour ne pas rester figés dans des organisations dépassées ». Les discours recueillis en entretiens sur le niveau d'auto-efficacité montrent que les directeurs des soins disposent, en général, d'une auto-efficacité de niveau plus élevé que celle des médecins inspecteurs de santé publique.

La deuxième phase de la recherche allait mesurer l'auto-efficacité de la quasi totalité de la population en formation à l'EHESP la même année, ce qui allait mettre en perspective les 
représentations et les discours sur l'auto-efficacité recueillis précédemment et auprès de quelques individus dans une phase exploratoire.

Le score moyen des 697 participants est de 29,13 points. Le score médian est de 29 points, le mode de 30 points et l'écart-type est de 3,64.

Nous avons pu mettre en évidence que le niveau d'auto-efficacité est indépendant de l'âge des cadres et dirigeants et du nombre d'années d'expérience professionnelle. En revanche, le sexe et le niveau d'étude initial influencent relativement le niveau d'auto-efficacité, tout comme la complexité perçue de la fonction. Les hommes sont significativement plus auto-efficaces que les femmes si l'on compare les moyennes des modalités avec le test de Student (au risque de 95\%) $: \mathrm{t}=3,03,1-\mathrm{p}=99,7 \%$. Ces résultats sont inattendus si nous comparons nos données avec celles de l'étude sur les apprentissages professionnels informels où les auteurs notent que les variables descriptives (sexe, âge, ancienneté, catégorie socioprofessionnelle) ne sont pas liées à l'auto-efficacité (FOLLENFANT A., MEYER T., 2003). L'indépendance des variables serait conforme avec l'idée que l'auto-efficacité est une «variable médiatrice, autonome par rapport aux déterminants sociologiques classiques » (CARRÉ P., 2003).

Il est intéressant de constater que ce ne sont pas les plus diplômés qui sont les plus autoefficaces, mais ceux qui ne sont titulaires que du baccalauréat: $\mathrm{t}=3,64,1-\mathrm{p}=>99.99 \%$. Parmi les élèves en formation à l'EHESP, les directeurs des soins sont aussi les moins diplômés. Ils ont bénéficié de promotions professionnelles et non universitaires jusqu'à la réussite du concours national. Nous allons le constater en nous intéressant au score moyen par filière, les directeurs des soins sont significativement plus auto-efficaces que les onze autres filières en formation statutaire ainsi que le montre le graphique suivant. Les abréviations des filières sont les suivantes : Médecins territoriaux (MT), directeurs d'établissement sanitaire et social (D3S), ingénieurs du génie sanitaire (IGS), médecins inspecteurs de santé publique (MISP), médecins inspecteurs régionaux du travail (MIRTMO), inspecteurs de l'action sanitaire et sociale (IASS) et inspecteurs principaux (IPASS), médecins de l'éducation nationale (MEN), ingénieurs d'études sanitaires (IES), cadres A détachés, directeurs d'hôpital (DH), directeurs des soins (DS). 


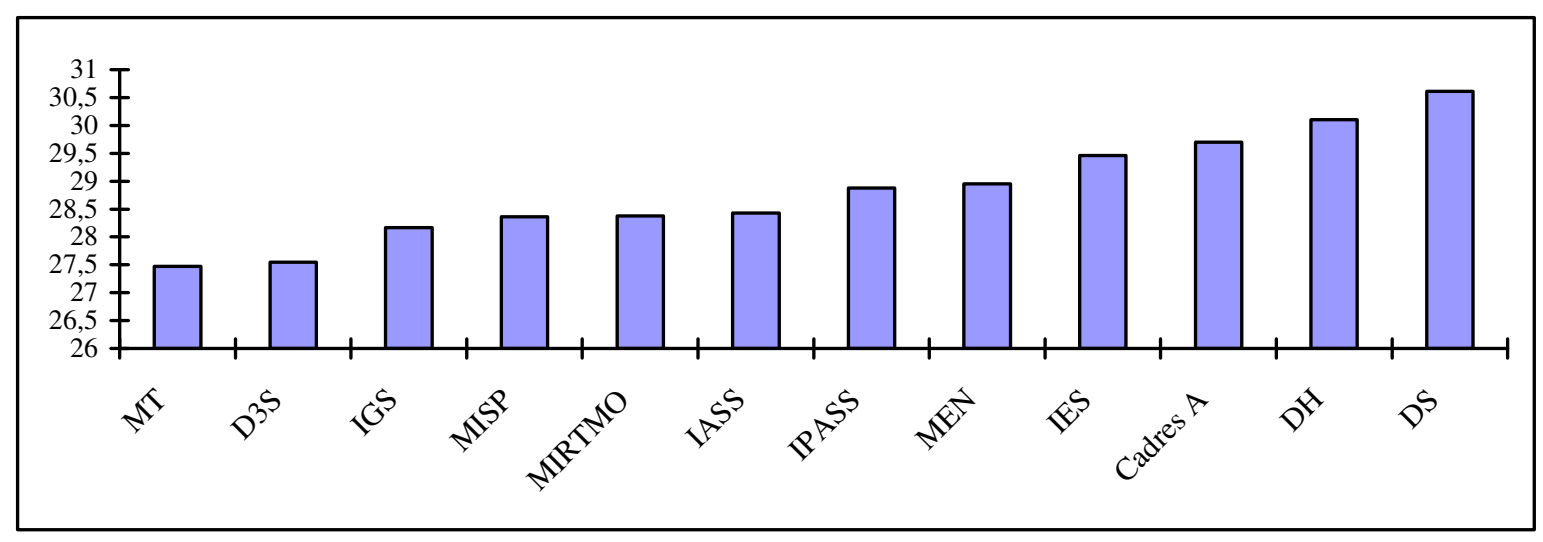

Les promotions appartenant aux filières de gestion d'établissements hospitaliers obtiennent des scores plus élevés : DS et DH à l'exception des D3S. Les filières d'inspecteurs de l'action sanitaire et sociale (IASS) et de médecins, se positionnent sur des scores plus faibles, voire significativement bas comme les médecins territoriaux. Ainsi, les «décideurs » auraient un score plus élevé que ceux qui occupent une fonction de «conseil ». En effet, le clivage ne se ferait pas entre filières hospitalières (DH, D3S, DS) et filières «État » (IASS, médecins, etc.) mais entre les directeurs (DH, DS, Cadres A détachés, D3S) qui possèderaient plus de moyens efficaces pour donner suite à leurs décisions et les «conseilleurs », les «régulateurs de l'action publique », ne disposant pas toujours des moyens de faire reconnaître leur expertise, sur les terrains ou au ministère.

Il restait à comprendre le rôle potentiel joué par l'analyse de l'activité dans le développement de l'auto-efficacité et s'assurer que l'auto-efficacité pouvait encore être augmentée dans le cadre du dispositif décrit supra. Pendant la période, le niveau d'auto-efficacité de l'ensemble de la promotion a légèrement augmenté, mais les directeurs des soins qui ont bénéficié d'une séquence d'analyse de l'activité ont vu leur niveau d'auto-efficacité augmenté trois fois plus que celui du groupe contrôle. Cette progression est significative et concerne tout particulièrement les sujets du groupe test qui exprimaient une auto-efficacité plus faible, + 8,44 points en moyenne sur une échelle de 30 points. Le test de Wilcoxon indique que la période a eu un effet sur le groupe test et nous pouvons penser que cet effet est créé par le dispositif d'analyse de l'activité. Si Tc $=\min (\mathrm{D}+, \mathrm{D}-)<\mathrm{T}$ théorique, rejet de $\mathrm{H} 0$ (hypothèse 
H0 : la période n'a pas d'effet), or Tc min étant égal à 24,5 et il est inférieur à T théorique, égal ici à 30 .

Les directeurs des soins de faible niveau initial du groupe contrôle n'augmentent que de 0,82 point. Les directeurs des soins les plus auto-efficaces du groupe test et du groupe contrôle ont un score moyen qui reste stable, voire qui diminue très légèrement de 0,17 point. Proches du maximum de l'échelle, pouvaient-ils encore augmenter leurs score?

Les directeurs des soins au score intermédiaire (groupe contrôle) connaissent une progression sensible de 2,50 points, en dehors de tout dispositif expérimental d'analyse de l'activité. L'analyse montre que les participants inclus dans ce groupe montraient une attitude proactive d'engagement en formation marquée par la recherche spontanée des effets attendus par l'analyse de l'activité. A la différence du sous-groupe test de niveau faible, ces participants disposaient déjà des ressources cognitives liées à un niveau intermédiaire d'auto-efficacité. Ces deux facteurs, engagement en formation et niveau intermédiaire d'auto-efficacité, paraissent liés pour expliquer l'évolution de leur score.

Après vérification statistique, nous pouvons conclure que l'analyse de l'activité a eu un effet sur le groupe test et, plus précisément, sur les personnes de faible niveau initial d'autoefficacité.

\section{Discussion}

Plusieurs résultats sont à retenir de cette recherche. Tout d'abord, nous avons produit une série de données tout à fait nouvelles sur la distribution des scores d'auto-efficacité au sein d'une population de cadres et de dirigeants de la santé publique. Au-delà, le résultat principal de cette recherche est d'établir une corrélation entre la participation à un dispositif formel ou informel d'analyse de l'activité et la construction de l'auto-efficacité au travail. Cherchant à expliquer ces résultats, nous estimons que l'analyse de l'activité est efficace parce qu'elle contribue à générer un processus de généralisation et de reconstruction de sens, d'augmentation du niveau d'abstraction dans la compréhension des comportements et d'attribution des actes et des résultats à soi-même. Il s'agit vraisemblablement des facteurs du développement de l'auto-efficacité en situation d'analyse de l'activité. Parce que ces facteurs agissent aussi au sein du sous-groupe contrôle de niveau intermédiaire, nous pouvons 
comprendre l'augmentation, plus modérée, de leurs scores d'auto-efficacité. Ces participants enclenchent spontanément une telle démarche autoformative et ils parviennent à des résultats en dehors d'un dispositif expérimental ou institué d'analyse de l'activité.

Nous le savions déjà avec les travaux de Pierre Pastré : l'analyse de l'activité du point de vue de la didactique professionnelle provoque des effets de genèse conceptuelle, de conceptualisation dans l'action. Encore faudrait-il cibler finement les publics et individualiser les parcours de formation pour rechercher les effets maximum de conceptualisation dans l'action et d'augmentation de l'auto-efficacité. Nous le constatons avec cette recherche : un dispositif de formation peut poursuivre des objectifs d'acquisition de compétences et de conceptualisation dans l'action, il ne provoquera pas automatiquement un développement de l'agentivité des apprenants. Finalement, cette recherche renouvelle la réflexion sur l'interaction entre un dispositif de formation, notamment le rôle d'autrui dans la construction de l'auto-efficacité au travail, et les dynamiques autoformatives, faisant de l'être humain un sujet en devenir capable de s'auto-développer.

En fait, la variable clé se trouve être l'auto-efficacité. Ce facteur représente une disposition cognitive propre à chaque apprenant mais il est également sensible à une action appropriée de l'ingénierie pédagogique. Nous retiendrons que si des individus bénéficient d'un dispositif d'analyse de l'activité et que leur niveau d'auto-efficacité augmente simultanément, ils réunissent tout à la fois la compétence effective et le sentiment de compétence, ressources pour dynamiser leur parcours professionnel.

Nos conclusions resteront toutefois limitées par crainte des effets potentiels des biais entrevus dans le dispositif. Nous citerons, par exemple, les biais d'attribution causale et d'autoprésentation de soi, voire le biais de Hawthorne ou celui de régression vers la moyenne. Tous ont pu influer, même dans une proportion variable, sur les résultats.

\section{Conclusion}

Nous nous sommes intéressé aux transformations qui affectent conjointement l'activité et les individus qui agissent. Dans le champ du travail, cette question est traitée par une grande variété d'approches. Souvent, elles tendent à montrer que l'individu se transforme par son expérience, par son activité antérieure dont il a tiré profit. Certes, l'activité constructive 
accompagne l'activité productive (RABARDEL P., 2005). Le couplage entre schème et situations est indispensable à considérer, pour autant, il faudrait se garder de penser l'activité comme un processus désincarné, modélisable en dehors des individus qui agissent et font preuve d'agentivité. Pourquoi agissent-ils et quels sont les ressorts du comportement performant? A chaque instant, l'individu traite de l'information, sélectionne les stimulations de l'environnement et organise ses conduites. Ses modèles cognitifs et opératifs se structurent progressivement. Mais surtout, une liberté de choix se manifeste qui amène à agir, ou ne pas agir, au gré de l'évaluation des variables situationnelles, des capacités du moment et de la valeur accordée aux performances obtenues. Nous possédons une capacité interne à diriger notre vie sans pour autant faire abstraction des influences externes. Le modèle du sujet agentique intègre la faculté de pouvoir systématiquement négocier ses comportements, ses relations affectives et ses actions avec son environnement physique ou social. Il dispose d'une auto-efficacité qui médiatise l'ensemble de ces influences et de ces déterminants. L'autoefficacité oriente les comportements et constitue une ressource pour leur autorégulation. En cela, l'auto-efficacité se révèle un bon prédicteur, voir un précurseur, des comportements efficaces. L'individu est en même temps produit et producteur de son environnement, et, en tout cas, de ses actions et des changements qu'il suscite.

Il apparaît ainsi que la conceptualisation dans l'action n'est pas une théorie de l'agentivité humaine, de même que la théorie socio cognitive n'est pas une théorie de l'activité. L'une s'attache à comprendre l'organisation cognitive de l'activité, l'autre considère la puissance d'agir des individus et des groupes sociaux dans une perspective développementale. Pour autant, comment comprendre l'agentivité et l'auto-efficacité sans s'intéresser à l'activité ?

Nous considérons la construction des compétences comme un espace potentiel de rencontre entre deux grands ensembles théoriques éprouvés mais qui ne conversent pas toujours. Cette recherche a provoqué cette convergence.

En prolongement de cette recherche, nous pensons que de nouvelles hypothèses pourraient être testées. Dans le champ du management hospitalier, nous observons que l'essentiel du travail consiste à traiter des tâches discrétionnaires. En effet, la prescription est de plus en plus large, générale et vague. Les buts fixés aux managers, aux directeurs des soins, sont remarquablement flous (par exemple: «garantir la qualité de la prise en charge des patients ») et les critères de réussite ne sont pas toujours formulés précisément. Les directeurs 
des soins ont donc très régulièrement le choix entre plusieurs stratégies d'action. Ils construisent leurs réponses et leurs actions sans pouvoir appliquer de quelconques démarches stéréotypées et standardisées. Ce type de contexte requiert peut-être plus de capacités d'adaptation que d'habiletés de reproduction à l'identique.

\section{Bibliographie}

BANDURA A. (2003). Auto-efficacité. Le sentiment d'efficacité personnelle. Bruxelles : De Boeck.

BANDURA A. (2005). "Growing centrality of self-regulation in health promotion and disease prevention ». The european health psychologist, septembre 2005 (1).

BEAUVOIS J-L., JOULE R-V. (1987). Petit traité de manipulation à l'usage des honnêtes gens. Grenoble : Presses Universitaires de Grenoble.

CARRÉ P. (2003). «Peut-on optimiser les apprentissages professionnels informels ? ». in Séminaire des responsables de formation - IRA Bastia, L'expérience est-elle formatrice ? Rennes : Réseau des écoles de service public.

CLOT Y., FAÏTA D., FERNANDEZ G., SCHELLER L. (2000). «Entretiens en autoconfrontation croisée : une méthode en clinique de l'activité ». Pistes, 2.

COULET J-C., CHAUVIGNÉ C. (2005). « Passer d'un référentiel de compétences à une ingénierie de formation ». Éducation Permanente, 165 (4).

ENLART S. (2007). "Compétences sociales et relationnelles et contextes professionnels». in THIBERGE B., La question des compétences sociales et relationnelles. Points de vue de praticiens. Paris : L'Harmattan.

ENLART S. (2008). L'assujettissement. Analyse des pratiques de GRH, formation et management dans la relation individu/organisation. HDR, Université Paris-Ouest Nanterre La Défense, Sciences de l'éducation, Nanterre.

FABRE M. (2006). « Analyse des pratiques et problématisation ». Recherche et formation, 51

FOLLENFANT A., MEYER T. (2003). «Pratiques déclarées, sentiment d'avoir appris et autoefficacité au travail. Résultats de l'enquête quantitative par questionnaires ». in CARRE P., CHARBONNIER O. (dir.), Les apprentissages professionnels informels. Paris : L'Harmattan.

FRANÇOIS P-H., BOTTEMAN A. (2002). "Théorie sociale cognitive de Bandura et bilan de compétences : applications, recherches et perspectives critiques ». Carriéologie. Revue francophone internationale, 8 (3-4).

GALAMBAUD B. (2002). Si la GRH était de la gestion. Paris : Éditions Liaisons.

JÉZEGOU A. (2005). Formations ouvertes. Libertés de choix et autodirection de l'apprenant. Paris : L'Harmattan.

KIESLER C. (1971). The psychology of commitment. New York : Academic Press.

LEPLAT J. (2000). L'analyse psychologique de l'activité en ergonomie. Toulouse : Octarès.

MÉTRAL J-F., MAYEN P. (2007). «Saisir les espaces de liberté des dispositifs pour proposer des situations de mise en activité ». Questions vives, 4 (8). 
NAGELS M., LE GOFF M. (2008). «Des référentiels de compétences innovants : quelle appropriation par les enseignants ?». in Télécom Bretagne, Actes du Veme colloque Questions de pédagogie dans l'enseignement supérieur. Enseigner, étudier dans le supérieur : pratiques pédagogiques et finalités éducatives. Brest : Télécom Bretagne, ENESIETA, École navale, Université de Bretagne occidentale.

NAGELS M., POURRIERE J-L. (2007). « Didactiser l'invisible ? Tutorat et professionnalisation des directeurs des soins en formation à l'École nationale de la santé publique ». in AECSE, Congrès AECSE. Actualité de la Recherche en Éducation et en Formation. Strasbourg.

OLRY P. (2004). «Saisir son expérience lors d'une VAE. L'auto-analyse du travail en perspective ». Éducation permanente, 159 (2).

PASTRÉ P. (2005a). «La conception de situations didactiques à la lumière de la théorie de la conceptualisation dans l'action ». in RABARDEL P., PASTRÉ P. (dir.), Modèles du sujet pour la conception. Dialectiques activités développement. Toulouse : Octarès.

PASTRÉ P. (2005b). «La deuxième vie de la didactique professionnelle ». Éducation Permanente, 165 (4).

PASTRÉ P., MAYEN P., VERGNAUD G. (2006). «La didactique professionnelle ». Revue française de pédagogie, 154

PY J., SOMAT A. (1991). «Normativité, conformité et clairvoyance : leurs effets sur le jugement évaluatif ». in BEAUVOIS J-L., JOULE, MONTEIL J-M., Perspectives cognitives et conduites sociales. Fribourg : Delval.

RABARDEL P. (2005). «Instrument subjectif et développement du pouvoir d'agir ». in RABARDEL P., PASTRÉ P. (dir.), Modèles du sujet pour la conception. Dialectiques activités développement. Toulouse : Octarès.

RICEUR P. (1986). Du texte à l'action. Paris : Le Seuil.

SIX-TOUCHARD B. (1998). «Développement de la compétence tutorale par l'auto-analyse du travail ». Éducation permanente, 135

SIX-TOUCHARD B., CARLIN N. (2003). «Impacts de l'auto-analyse sur les opérateurs et leur travail ». in Société d'Ergonomie de Langue Française, XXXVIIIème Congrès de la SELF. Paris.

THIBERGE B. (2007). La question des compétences sociales et relationnelles. Points de vue de praticiens. Paris : L'Harmattan.

VERGNAUD G. (1998). «Au fond de l'action, la conceptualisation ». in BARBIER J-M., Savoirs théoriques et savoirs d'action. Paris : PUF.

VERGNAUD G. (2006). «Une approche cognitive des compétences et de l'activité ». in Université de Bretagne, Compétences, emploi et enseignement supérieur. L'enseignement supérieur prépare-t-il à l'emploi ? Rennes. 